\title{
Study of mRNA turnover never decays
}

\author{
ANN-BIN SHYU \\ Department of Biochemistry and Molecular Biology, The University of Texas-Medical School, Houston, Texas 77030, USA
}

As I reflect on what the field of mRNA turnover has achieved over the past two decades, the first things that pop up in my mind are the significant recognition the field has attained and how far the field has advanced. I recall that in the early 1990s, mRNA degradation was viewed by many researchers as an uncontrolled scavenger process, much like a cellular trash can where cells dump old mRNAs that are no longer in use. Of course, without transcription there will be no gene expression in the first place. It is thus not surprising that, when dealing with problems of regulation of eukaryotic gene expression, researchers at the time intuitively placed regulation mainly at the level of transcription. This idea was prevalent even though degradation of mRNA had been shown to be one of the principal means by which prokaryotic genes are regulated. It was not until the mid 1990s that researchers started to appreciate that modulation of mRNA stability plays a critical role in regulating gene expression, allowing cells to change the levels of gene expression rapidly in response to a dynamic cellular environment.

In the early and mid 1990s, use of budding yeast and a transcriptional pulsing approach permitted investigators to chase a newly synthesized mRNA into its decay products in a genetically manipulated background. This work helped elucidate the mechanistic steps and basic constituents of mRNA turnover machinery. The development of siRNA-based gene knockdown technologies in the early 2000s greatly facilitated deciphering the mRNA decay pathways in mammalian cells. Cumulative evidence establishes that deadenylation, i.e., shortening of the $3^{\prime}$ poly $(\mathrm{A})$ tail, is the major means of triggering eukaryotic mRNA turnover. Unexpectedly, instead of being followed by $3^{\prime}$ to $5^{\prime}$ exonucleolytic digestion of the mRNA body, deadenylation primarily leads to removal of the $5^{\prime}$ cap of mRNA followed by rapid $5^{\prime}$ to $3^{\prime}$ digestion of the mRNA body. At first glance, this major route of mammalian mRNA decay may seem indirect and inefficient. However, it is clear now that this sequence of steps in mRNA decay serves important purposes. Decapping prevents additional loading of ribosomes at the same time allowing ribosomes already on the targeted mRNAs to finish translation without generating potentially cytotoxic truncated polypep-

Corresponding author: Ann-Bin.Shyu@uth.tmc.edu

Article and publication date are at http://www.rnajournal.org/cgi/doi/10. 1261/rna.050526.115. Freely available online through the RNA Open Access option. tide chains. This mRNA degradation sequence also adds another layer of regulation of mRNA function. Depending on their environment, cells can either reuse or rescue the poly(A) shortened mRNA for later translation or degrade that mRNA permanently. It is therefore not entirely surprising to find that microRNAs, after forming the RISC complex on target mRNAs, shunt those mRNAs to this major route of mRNA degradation and accelerate their deadenylation.

The discovery of GW/P-bodies in 2002-2003 brought much excitement to the mRNA turnover field and to the entire RNA biology field, and it drove much biological research in the next decade. GW/P-bodies are cytoplasmic foci of messenger ribonucleoprotein particle (mRNP) aggregates containing a plethora of translation repressors and mRNA decay factors. The information about mRNA decay pathways and participating factors that accumulated through the 1990s helped pave the way to understanding GW/P-bodies. Few could have imagined at the beginning that investigation of these cytoplasmic foci would eventually occupy hundreds of biologists. Although it has been suggested that GW/P-bodies function in translational control and mRNA degradation, their exact role(s) in regulating gene expression, and how their assembly and disassembly is controlled, remain challenging and critical issues in the RNA field.

Another important development in the mRNA turnover field that caught the attention of many biologists was the elucidation of how rapid decay of an mRNA is triggered by a nonsense or premature termination codon (PTC) in the open-reading-frame (ORF). This intriguing yet elusive problem of how eukaryotic cells discern whether the ORF of a mature mRNA is prematurely terminated and thus needs to be promptly degraded drew the efforts of many investigators starting in the late 1990s. These efforts led to a fairly thorough understanding of the mechanism of nonsense-mediated mRNA decay (NMD) and its manifestation in human genetic diseases. Several important principles regarding mRNA processing in the nucleus and its metabolism in the cytoplasm emerged. One principle is that the experience of an mRNA in the nucleus influences that mRNA's fate in the cytoplasm. As it turns out, after pre-mRNA splicing, a group of proteins, termed the exon-junction-complex (EJC), associate with

(c) 2015 Shyu This article, published in $R N A$, is available under a Creative Commons License (Attribution-NonCommercial 4.0 International), as described at http://creativecommons.org/licenses/by-nc/4.0/. 
mRNAs undergoing maturation in the nucleus. The EJC proteins help guide the pre-mRNA through subsequent processing steps and/or nuclear export. Some EJC constituents accompany mRNA to the cytoplasm, where they influence the translation, stability and localization of the mRNA. A second principle is that mRNAs fulfill their functions in the form of mRNPs. One prominent feature of mRNP complexes is that they are highly dynamic, with rapid exchanges of mRNP protein constituents dictating the mRNA functions and fate at each step during their metabolism. These alterations in mRNP protein composition are collectively termed mRNP remodeling. Any failure to appropriately remodel an mRNP complex has the potential to disrupt downstream events that determine the mRNA's fate, as exemplified by the NMD process. Thus, mRNPs represent highly dynamic, functional forms of mRNAs. A third principle is that many mRNP constituent proteins shuttle between the nucleus and cytoplasm to fulfill their functions in mRNA metabolism. These shuttling proteins usually live a double-life in eukaryotic cells, with one role in the nucleus and another in the cytoplasm, and disrupting the proteins' shuttling leads to formation of dysfunctional mRNPs.

The molecular constituents of individual mRNPs and their remodeling during the lifetime of mRNAs are exciting and relatively underexplored areas of research in RNA biology. With over 800 putative human RNA-binding proteins (RNA-BPs) and the many additional proteins that interact with the RNA-BPs, identifying the makeup of individual mRNPs and defining specific roles for individual RNP proteins are challenging but important tasks. Equally challenging is elucidation of the post-translational modification mechanisms that regulate $\mathrm{mRNP}$ remodeling, as these modifications could impact the fate of mRNAs in a transcript-spe- cific or transcriptome-wide manner. At this point, few signaling pathways associated with mRNP remodeling have been identified. A combination of RNA-affinity chromatography and quantitative proteomics may prove useful for characterizing modifications of the RNA-binding proteome.

The physiological importance of mRNA-BPs and mRNP complexes is underscored by their linkage to many human diseases. One recent example is a malfunction of hnRNPA1 that results in mRNP aggregation and is linked to neuronal degenerative diseases. There is also the instance of mutations in a protein segregase, $\mathrm{p} 97$, that have been genetically linked to inclusion body myopathy, Paget's disease, frontotemporal dementia, and amyotrophic lateral sclerosis. The molecular pathogenesis of these diseases remains poorly understood. It has been proposed that some severe neurodegenerative diseases associated with p97 mutations involve a defect in $m R N P$ remodeling that results in $\mathrm{mRNP}$ aggregation and aberrant mRNA translation. It is clear from recent studies that mammalian p97 is critical for autophagic clearance of stress granule-related and pathogenic RNP granules, which accumulate in p97-linked degenerative diseases. Studies of the regulation of mRNP remodeling may suggest ways to therapeutically manipulate a subset of mRNPs under particular physiological or pathological conditions.

It has recently become evident just how rapidly new techniques, such as proteome- and transcriptome-based technologies, can catch fire and spread to labs that don't specialize in methods development but nevertheless are able to rapidly adopt-and improve upon-these new approaches. In the next decade, we should expect to see a more comprehensive grasp of the makeup of individual mRNPs, of mRNP dynamics, and of the regulation of mRNP remodeling and its connections to mRNP-related human diseases. 

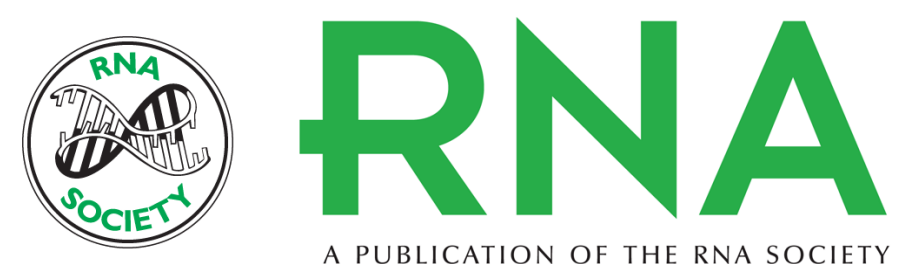

A PUBLICATION OF THE RNA SOCIETY

\title{
Study of mRNA turnover never decays
}

\author{
Ann-Bin Shyu
}

RNA 2015 21: 738-739
Open Access Freely available online through the RNA Open Access option.
Creative This article, published in $R N A$, is available under a Creative Commons License Commons (Attribution-NonCommercial 4.0 International), as described at License http://creativecommons.org/licenses/by-nc/4.0/. Email Alerting
Service

To subscribe to RNA go to:

http://rnajournal.cshlp.org/subscriptions 